\title{
Genome Sequence Resource for the Plant Pathogen Sclerotinia sclerotiorum WH6 Isolated in China
}

\author{
Xiong Zhang, Xiaohui Cheng, Lijiang Liu, ${ }^{\dagger}$ and Shengyi Liu
}

Key Laboratory of Biology and Genetic Improvement of Oil Crops, Ministry of Agriculture of the PRC, Oil Crops Research Institute, Chinese Academy of Agricultural Sciences, Wuhan 430062, China

\begin{abstract}
Sclerotinia sclerotiorum is a notorious fungal pathogen that causes sclerotinia stem rot (SSR) on many important crops in China and worldwide. Here, we present a high-quality genome assembly of $S$. sclerotiorum strain WH6 using the PacBio SMRT cell platform. The assembled genome has a total size of $38.96 \mathrm{Mbp}$, with a contig N50 length of 1.90 Mbp, and encodes 10,512 predicted coding genes, including 685 secreted proteins and 65 effector candidates. This is the first report of a S. sclerotiorum genome sequence from China. The WH6 genome sequence provides a valuable resource for facilitating our understanding of S. sclerotiorum-host interactions and SSR control in China and the world.
\end{abstract}

\section{Genome Announcement}

Sclerotinia sclerotiorum is a remarkable plant pathogenic fungus for its broad host range and destructive diseases. S. sclerotiorum can infect over 400 different hosts, including many economically important crops such as oilseed rape, soybean, pea, sunflower, and others (Bolton et al. 2006). Sclerotinia stem rot (SSR), caused by S. sclerotium, is one of the most serious diseases on oilseed rape and soybean crops in China (Wang et al. 2014) and the U.S. (Bolton et al. 2006). S. sclerotiorum can cause up to $80 \%$ yield loss in individual oilseed rape fields during serious outbreaks (Li et al. 2006). Until now, complete genome sequence has been available only for S. sclerotiorum 1980 UF-70 from the U.S. (Amselem et al. 2011; Derbyshire et al. 2017). The genome sequences of $S$. sclerotiorum strains from China remain to be determined and the genomic difference between Chinese strains and other strains are still unclear.

In this study, we sequenced the genome of S. sclerotiorum WH6, which was isolated from a diseased rapeseed plant in our experimental field station of Hubei Province, China. The genomic DNA of the strain was extracted and sequenced using long-read PacBio platforms with single-molecule real-time (SMRT) technology (Beijing Grandomics Biosciences Co., Ltd.) with an average insert size of $20 \mathrm{~kb}$. In total, 774,934 long reads, representing 166× coverage of the $S$. sclerotiorum genome, were generated after sequencing. Sequence filtering and de novo assembling were performed on full PacBio long reads using Canu v1.7.1 (Koren et al. 2017). The final genome assembly comprised $38.96 \mathrm{Mbp}$ containing 28 contigs, with an N50 length of $1.90 \mathrm{Mbp}$ and a GC content of $41.57 \%$. The longest contig consisted of $3.95 \mathrm{Mbp}$. The genome of S. sclerotiorum WH6 was similar to the genome of S. sclerotiorum 1980 UF-70 (Derbyshire et al. 2017), which was $38.91 \mathrm{Mbp}$ long with $41.56 \%$ GC content. These and other genome annotation statistics of 980 UF-70 were shown in Table 1. Completeness of the WH6 assembly was assessed using BUSCO v4.1.4 with the lineage-specific dataset helotiales_odb10 (Seppey et al. 2019). This analysis indicated that the genome assembly displayed a high overall completeness of $97.89 \%$, only $0.33 \%$ of BUSCOs were present as
Funding

This project was supported by the Central Public-interest Scientific Institution Basal Research Fund (Y2020YJ03, 1610172021001); the China Agriculture Research System of MOF and MARA (CAAS-12) and the Agricultural Science and Technology Innovation Program of the Chinese Academy of Agricultural Sciences (CAAS-ASTIP-2013-OCRI).

\section{Keywords}

fungi, disease management, oilseeds and legumes

${ }^{\dagger}$ Corresponding author: L. J. Liu; liulijiang @ caas.cn

The author(s) declare no conflict of interest.

Accepted for publication 30 March 2021.

(C) 2021 The American Phytopathological Society 
Table 1. Summary of Sclerotinia sclerotiorum WH6 genome assembly and comparison with strain 1980 UF-70

\begin{tabular}{lcc} 
Genomic features & WH6 & $\mathbf{1 9 8 0 ~ U F - 7 0 ~}$ \\
Sequencing platform & PacBio RSII & PacBio \\
Coverage $(x)$ & 166 & 36 \\
Assembly size (bp) & $38,957,821$ & $38,906,497$ \\
Number of contigs & 28 & 17 \\
GC content (\%) & 41.57 & 41.56 \\
N50 length (Mbp) & $1,900,497$ & $2,387,400$ \\
BUSCO completeness (\%) & 97.89 & $97.78^{\mathrm{a}}$ \\
Repeat rate (\%) & 11.99 & 12.96 \\
Predicated protein-coding genes & 10,597 & 11,130 \\
Annotated proteins with the nonredundant database & 10,512 & $10,802^{\mathrm{a}}$ \\
Annotated proteins with Swiss-prot & 6,375 & $6,401^{\mathrm{a}}$ \\
Secreted proteins & 685 & $711^{\mathrm{b}}$ \\
Candidate effectors & 65 & $70^{\mathrm{b}}$ \\
Reference & This study & Derbyshire et al. 2017 \\
\hline
\end{tabular}

\footnotetext{
a These data were not described in the original study and identified by the same pipeline (this study).

${ }^{b}$ These data were described in the original study and reanalyzed by the same pipeline (this study) to ensure a fair comparison.
}

fragments, and $1.78 \%$ were missing. Repeat sequences of the genome were carried out by RepeatMasker v4.0.7 (http://www.repeatmasker.org) using a de novo repeats library generated by Repeat Modeler pipeline v1.0.7 (Saha et al. 2008). As a result, approximately $11.99 \%$ of sequences were identified as repeat sequences.

After repeat-masking, exonerate v2.2.0 (Slater and Birney 2005) and BLAST v2.2.28 (Camacho et al. 2009) were run within Maker for evidence-based alignments and Augustus v3.2.2 (Keller et al. 2011) and SNAP v2006-07-28 (Korf 2004) for ab initio gene prediction. Overall, 10,597 protein-coding genes were identified, with an average length of 1,400 bp, constituting $38.07 \%$ of the total genome. Among the predicated genes, 10,512 were mapped to the National Center for Biotechnology Information nonredundant protein database, 6,375 were mapped to the Swiss-Prot database, 5,487 were assigned GO terms, and 3,100 were mapped to the KEGG database, respectively. After analysis with SignalP v4.1 (Petersen et al. 2011) and THHMM v2.0 (Krogh et al. 2001), a total of 685 secreted proteins were identified. Among the secreted proteins predicted, 65 proteins were identified as effector candidates by EffectorP v2.0 (Sperschneider et al. 2018). In addition, 603 putative carbohydrate-active enzymes, 2,218 transporters, 115 kinases, and 3,487 pathogen-host interaction proteins were predicted using the CAZy (Cantarel et al. 2009), TCDB (Saier et al. 2006), Kinomer (Martin et al. 2009), and $\mathrm{PHI}$ databases (Urban et al. 2020) ( $E$ values were all $<1 \mathrm{e}-5$ ), respectively.

In summary, we reported a high-quality genome assembly of S. sclerotiorum strain WH6. Our data provides a new resource to understand the diversity of the $S$. sclerotiorum populations worldwide and the underlying mechanisms of potential pathogenicity differentiation among different S. sclerotiorum strains and contributes to SSR control in China and the world. The genome assembly and associated raw data have been deposited into CNGB Sequence Archive (CNSA) of the China National GeneBank Database (CNGBdb) (Chen et al. 2020) with accession number CNP0001567 (https://db.cngb.org/cnsa/project/CNP0001567/ reviewlink/).

\section{Literature Cited}

Amselem, J., Cuomo, C. A., van Kan, J. A., Viaud, M., Benito, E. P., Couloux, A., Coutinho, P. M., de Vries, R. P., Dyer, P. S., Fillinger, S., Fournier, E., Gout, L., Hahn, M., Kohn, L., Lapalu, N., Plummer, K. M., Pradier, J. M., Quevillon, E., Sharon, A., Simon, A., ten Have, A., Tudzynski, B., Tudzynski, P., Wincker, P., Andrew, M., Anthouard, V., Beever, R. E., Beffa, R., Benoit, I., Bouzid, O., Brault, B., Chen, Z., Choquer, M., Collemare, J., Cotton, P., Danchin, E. G., Da Silva, C., Gautier, A., Giraud, C., Giraud, T., Gonzalez, C., Grossetete, S., Guldener, U., Henrissat, B., Howlett, B. J., Kodira, C., Kretschmer, M., Lappartient, A., Leroch, M., Levis, C., Mauceli, E., Neuveglise, C., Oeser, B., Pearson, M., Poulain, J., Poussereau, N., Quesneville, H., Rascle, C.,
Schumacher, J., Segurens, B., Sexton, A., Silva, E., Sirven, C., Soanes, D. M., Talbot, N. J., Templeton, M., Yandava, C., Yarden, O., Zeng, Q., Rollins, J. A., Lebrun, M. H., and Dickman, M. 2011. Genomic analysis of the necrotrophic fungal pathogens Sclerotinia sclerotiorum and Botrytis cinerea. PLoS Genet. 7: e1002230.

Bolton, M. D., Thomma, B. P. H. J., and Nelson, B. D. 2006. Sclerotinia sclerotiorum (Lib.) de Bary: biology and molecular traits of a cosmopolitan pathogen. Mol. Plant Pathol. 7:1-16.

Camacho, C., Coulouris, G., Avagyan, V., Ma, N., Papadopoulos, J., Bealer, K., and Madden, T. L. 2009. BLAST plus: architecture and applications. BMC Bioinformatics $10: 421$ 
Cantarel, B. L., Coutinho, P. M., Rancurel, C., Bernard, T., Lombard, V., and Henrissat, B. 2009. The Carbohydrate-Active EnZymes database (CAZy): an expert resource for Glycogenomics. Nucleic Acids Res. 37:D233-D238.

Chen, F. Z., You, L. J., Yang, F., Wang, L. N., Guo, X. Q., Gao, F., Hua, C., Tan, C., Fang, L., Shan, R. Q., Zeng, W. J., Wang, B., Wang, R., Xu, X., and Wei, X. F. 2020. CNGBdb: China National GeneBank DataBase. Hereditas 42:799-809.

Derbyshire, M., Denton-Giles, M., Hegedus, D., Seifbarghy, S., Rollins, J., van Kan, J., Seidl, M. F., Faino, L., Mbengue, M., Navaud, O., Raffaele, S., HammondKosack, K., Heard, S., and Oliver, R. 2017. The complete genome sequence of the phytopathogenic fungus Sclerotinia sclerotiorum reveals insights into the genome architecture of broad host range pathogens. Genome Biol. Evol. 9:593-618.

Keller, O., Kollmar, M., Stanke, M., and Waack, S. 2011. A novel hybrid gene prediction method employing protein multiple sequence alignments. Bioinformatics 27:757-763.

Koren, S., Walenz, B. P., Berlin, K., Miller, J. R., Bergman, N. H., and Phillippy, A. M. 2017. Canu: scalable and accurate long-read assembly via adaptive k-mer weighting and repeat separation. Genome Res. 27:722-736.

Korf, I. 2004. Gene finding in novel genomes. BMC Bioinformatics 5:59.

Krogh, A., Larsson, B., von Heijne, G., and Sonnhammer, E. L. L. 2001. Predicting transmembrane protein topology with a hidden Markov model: Application to complete genomes. J. Mol. Biol. 305:567-580.

Li, G. Q., Huang, H. C., Miao, H. J., Erickson, R. S., Jiang, D. H., and Xiao, Y. N. 2006. Biological control of sclerotinia diseases of rapeseed by aerial applications of the mycoparasite Coniothyrium minitans. Eur. J. Plant Pathol. 114: 345-355.
Martin, D. M., Miranda-Saavedra, D., and Barton, G. J. 2009. Kinomer v. 1.0: a database of systematically classified eukaryotic protein kinases. Nucleic Acids Res. 37:D244-D250.

Petersen, T. N., Brunak, S., von Heijne, G., and Nielsen, H. 2011. SignalP 4.0: discriminating signal peptides from transmembrane regions. Nat. Methods 8:785786.

Saha, S., Bridges, S., Magbanua, Z. V., and Peterson, D. G. 2008. Empirical comparison of ab initio repeat finding programs. Nucleic Acids Res. 36:2284-2294.

Saier, M. H., Jr., Tran, C. V., and Barabote, R. D. 2006. TCDB: the transporter classification database for membrane transport protein analyses and information. Nucleic Acids Res. 34:D181-D186.

Seppey, M., Manni, M., and Zdobnov, E. M. 2019. BUSCO: Assessing genome assembly and annotation completeness. Methods Mol. Biol. 1962:227-245.

Slater, G. S. C., and Birney, E. 2005. Automated generation of heuristics for biological sequence comparison. BMC Bioinformatics 6:31.

Sperschneider, J., Dodds, P. N., Gardiner, D. M., Singh, K. B., and Taylor, J. M. 2018. Improved prediction of fungal effector proteins from secretomes with EffectorP 2.0. Mol. Plant Pathol. 19:2094-2110.

Urban, M., Cuzick, A., Seager, J., Wood, V., Rutherford, K., Venkatesh, S. Y., De Silva, N., Martinez, M. C., Pedro, H., Yates, A. D., Hassani-Pak, K., and Hammond-Kosack, K. E. 2020. PHI-base: the pathogen-host interactions database. Nucleic Acids Res. 48:D613-D620.

Wang, Y., Hou, Y. P., Chen, C. J., and Zhou, M. G. 2014. Detection of resistance in Sclerotinia sclerotiorum to carbendazim and dimethachlon in Jiangsu Province of China. Australas. Plant Pathol. 43:307-312. 